\title{
A Positron Annihilation Study of Carbon Black and Carbon-Black-Filled Polybutadiene
}

\author{
D. H. D. West ${ }^{\star}$, V. J. McBrierty, and C. F. G. Delaney \\ Physical Laboratory, Trinity College, University of Dublin, Dublin 2, Ireland
}

Received 16 January 1978/Accepted 21 August 1978

\begin{abstract}
Studies have been made on positron lifetimes in carbon blacks and carbon black filled polybutadiene. The results for the carbon blacks can be interpreted with the aid of the theoretical results of Brandt and Paulin: Phys. Rev. B5, 2430 (1972) and show that while positron annihilation occurs principally from a bound state, diffusion in the carbon black is extremely small. A numerical upper limit on the diffusion coefficient has been obtained. The data from the rubber is discussed with the aid of a specially developed extension of the previous theory. Here one finds that whereas the variations in the long-component intensity with carbon black loading can be very satisfactorily explained, there is again no evidence for any diffusion effects in the rubber, and an upper limit can be placed on the diffusion coefficient here also. Indeed the variation in the long-component intensity with particle size goes the opposite way to what one would expect if diffusion were dominant. This effect is attributed to the increase in the amount of bound rubber as the particle size is reduced, and the consequent increase in the number of annihilation sites.
\end{abstract}

PACS: 78.70

The physical behaviour of non-crystalline polymers above their glass transition may be considerably modified by the incorporation of a reinforcing filler such as carbon black. Despite extensive investigation of reinforcement, motivated by its great technological importance, understanding of it is still largely phenomenological, and recent reviews [1-6] emphasize the multiplicity and complexity of the processes involved. These systems, and indeed carbon black itself, are ideally suited for study by the positron lifetime technique, in view of their interesting structure and free radical content.

One characteristic of the polymer which is known to be modified near the filler surface is the degree of motion which the molecules achieve, because a chain is effectively immobilized at a point of adsorption on a particle. Evidence of such immobilization has been found in NMR studies [7-11], and also in thermal expansion measurements [12]. In two of the NMR studies $[7,10]$, at least two regions of differing mobility

\footnotetext{
* Now at Randall Laboratory of Physics, University of Michigan, 500 East University, Ann Arbor, MI 48109, USA.
}

were found in bound rubber from which the unbound rubber had been extracted with a solvent. Possible molecular configurations in bound rubber have been discussed by several authors $[8,10,13]$.

The above-mentioned experiments typically yield a length which characterizes the approach of the mean value of the polymer property concerned (for example, molecular motion) to its asymptotic value remote from the filler surface. In the absence of more detailed information, the customary description of this length as a layer thickness should not be taken to imply any unusually large gradient of the polymer property at this distance from the filler surface.

\section{Experimental Procedure}

The positron lifetime spectrometer is as described previously [14], except that its time resolution, measured with ${ }^{60} \mathrm{Co}$, has been improved to 400 ps FWHM. The positron source was $5 \mu \mathrm{Ci}$ of ${ }^{22} \mathrm{Na}$ in a mica and nickel foil sandwich. RCA 8575 photomultipliers and NE 111 scintillators were employed, and timing signals 
Table 1. Properties of the carbon blacks

\begin{tabular}{lllclc}
\hline Trade Name & Type & EM diam, & $\begin{array}{l}N_{2}(\mathrm{BET}) \text { area } \\
{\left[\mathrm{m}^{2} / \mathrm{g}\right]}\end{array}$ & $\begin{array}{l}10^{-19} \times \text { spin } \\
\text { concentration } \\
{[\text { spins/g] }}\end{array}$ & $\begin{array}{c}N_{2} \text { diam. } \\
{[\mathrm{nm}]}\end{array}$ \\
\hline Regal & SRF & 75 & 30 & 4 & 100 \\
Statex G & GPF & 54 & 29 & 48 & 103 \\
Philblack A & FEF & 38 & 43 & 8 & 70 \\
Statex R & HAF & 24 & 84 & 5 & 29 \\
Vulcan 6 & ISAF & 22 & 104 & 5 & 29 \\
\hline
\end{tabular}

Table 2. Characteristics of the filled rubber samples

\begin{tabular}{llccc}
\hline $\begin{array}{l}\text { Sample } \\
\text { code }\end{array}$ & $\begin{array}{l}\text { Black } \\
\text { type }\end{array}$ & $\begin{array}{l}\text { Loading } \\
\text { [phr] }\end{array}$ & Millings & $\begin{array}{l}10^{-14}(b-a)^{-2} \\
(\text { see text) } \\
{\left[\mathrm{m}^{-2}\right]}\end{array}$ \\
\hline A & GPF & 100 & 30 & 21.7 \\
B & GPF & 80 & 30 & 15.8 \\
C & GPF & 60 & 30 & 10.6 \\
D & GPF & 40 & 30 & 6.3 \\
E & GPF & 20 & 30 & 2.8 \\
F & SRF & 50 & 40 & 8.8 \\
G & GPF & 50 & 40 & 8.3 \\
H & FEF & 50 & 40 & 18.0 \\
I & HAF & 50 & 40 & 68.1 \\
J & ISAF & 50 & 40 & 105 \\
\hline
\end{tabular}

derived using Ortec 270 constant fraction discriminators were applied to an Ortec 457 time to pulse height converter. Energy windows were set at $0.65-1.3 \mathrm{MeV}$ for the start channel and 0.15 to $0.5 \mathrm{MeV}$ for the stop channel. The start pulses were delayed and gated by the stop pulses to reduce the dead time of the converter. The lifetime spectra were analysed by the method of weighted least squares into two "sample" components, one "source" component and a constant background [15]. Each component consisted of a decaying truncated exponential folded with the measured resolution function.

Positron measurements were made on a) five different types of carbon black, b) filled rubber as a function of proportion of black for a single black type, and c) filled rubber as a function of black type for a fixed proportion of black.

Some properties of the carbon blacks used are listed in Table 1. EM particle diameters were measured with an Electron Microscope, the specific surface areas were determined by nitrogen absorption (BET method) and the spin concentrations were determined by paramagnetic resonance [16]. The rubber was cispolybutadiene (Shell Cariflex BR 1220) of density $1.01 \mathrm{~g} \mathrm{~cm}^{-3}$. The characteristics of the filled rubber samples are shown in Table 2 , in which the proportion of carbon black is given in p.h.r. (parts per hundred of rubber, by weight). The samples include some of those used in a previously cited NMR study [10] in which fuller details of sample preparation are given. Samples $\mathrm{A}-\mathrm{E}$ are identical to the corresponding ones in [10], and F-J differ from their counterparts in [10] only in having been milled an additional ten times during preparation. The filled samples were prepared several months before measurements were made, to allow the bound rubber to approach its limiting value [1]. Measurements on the filled rubber samples were made with the positron source sandwiched between two $2 \mathrm{~cm} \times 2 \mathrm{~cm} \times 2 \mathrm{~mm}$ pieces of the material concerned.

\section{Results and Discussion}

The results of the measurements are shown in Table 3, in which all quoted errors are one standard deviation, computed from the counting statistics only. (Note that $\tau_{1}$ is in ps and $\tau_{2}$ in ns.) We start with a discussion of the carbon black results.

\subsection{Carbon Black}

These results can be interpreted with the aid of the theoretical work of Brandt and Paulin [17] on combined positron diffusion and annihilation. They consider positrons thermalizing in a target consisting of small solids (spheres, cylinders or foils) interspersed with voids in which positrons are not stopped, (but into which they can diffuse as positronium) and obtain an expression for the fraction of particles $n(t)$ remaining at a time $t$ after entry, which exhibits the time spectrum explicitly. Their result for spherical particles is

$$
\begin{aligned}
n(t)= & 6 \sum_{\nu=1}^{\infty}\left(\frac{1}{\lambda_{v}^{2}}-\frac{\beta^{2}}{\lambda_{v}^{2} \beta^{2}+g^{2}}\right) \\
& \cdot \exp \left[\left(-\lambda_{\nu}^{2} \beta^{2}+1\right) \gamma_{s} t\right]+\Phi(\beta, g) \mathrm{e}^{-\gamma_{v} t},
\end{aligned}
$$

where

$\Phi(\beta, g)=6 \sum_{\nu=1}^{\infty} \frac{\beta^{2}}{\lambda_{v}^{2} \beta^{2}+g^{2}}$, 
$\gamma_{s}$ and $\gamma_{v}$ are the annihilation rates in the solids and the voids respectively, $g^{2}=1-\gamma_{v} / \gamma_{s}, \quad \beta^{2}=D /\left(\gamma_{s} R^{2}\right)$ and $D$ is the diffusion coefficient in the solids. $\Phi(\beta, g)$ $=3 \beta[\operatorname{coth}(g / \beta)-\beta / g] / g, \lambda_{v}=\pi v$ and $R$ is the radius of each solid. Using these results and our experimental data we now discuss the formation of positronium in carbon black and the possibility of its subsequent diffusion.

If positronium is formed in carbon black, (1) shows that if it diffuses out of the black particles this should give rise to a long component of lifetime $\gamma_{v}^{-1}=140 \mathrm{~ns}$ (i.e. the orthopositronium lifetime) in the time spectrum. In fact the carbon black spectra were adequately fitted by a single short component as tabulated, plus a constant background of $\approx 2.4$ counts/channel. If positronium is formed with probability $p$, then the relative intensity of the resulting long component would be $3 p \Phi / 4 \approx 9 p \beta / 4 g$ since $\Phi$ is small. If the observed long component comprises at most a fraction $r$ of all annihilations, then $r=9 p \beta / 4 g$ and substituting for $\beta$ and $g$, the radial diffusion coefficient $D$ must satisfy

$D<0.2 R^{2}\left(\gamma_{s}-\gamma_{v}\right) r^{2} / p^{2}$.

Attribution of the whole of the calculated background to the long component would give $r=0.04$, but the true background is observable in the time spectrum on one side of the peak, and from it one can reasonably infer that $r=0.01$.

A value for $p$ is more difficult to obtain. Apart from the present work, the only experimental evidence seems to be that of Berko [18], who observed the energy spectrum of the annihilation gammas with an $\mathrm{NaI}(T)$ detector, and found no perceptible filling-in of the minimum between the $0.51 \mathrm{MeV}$ photopeak and Compton edge, such as would be produced by 3gamma annihilation. This observation is however, also quite consistent with rapid pickoff. For free delocalised positrons in carbon black, the Dirac formula gives a lifetime of $334 \mathrm{ps}$ (excluding atomic core contributions), but this will be reduced by the polarisation of the electron distribution by the charge of the positron. This effect can be estimated from the theory of Brandt and Reinheimer for impurity screening in amorphous semiconductors [19]. Using the band-gap parameter appropriate for graphite in their closely related theory for crystalline semiconductors [20] (which predicts positron annihilation rates within $10 \%$ of the observed values for $\mathrm{Si}$ and $\mathrm{Ge}$ ), but setting the amorphicity to 1 and using the valence electron density appropriate for carbon black, one finds an electron density enhancement factor at the positron of 2.89 , which would reduce the free positron lifetime to $116 \mathrm{ps}$. This result is insensitive to the assumed band-gap value, and Brandt and Reinheimer [19] cited evidence that the observed bandgap is usually insensitive to the crystalline- amorphous transition. The band structure of carbon black is unusual and poorly understood [21], but this seems unlikely to lengthen the free positron lifetime by a factor of four. The observed $400 \mathrm{ps}$ lifetime must therefore be attributed not to free positrons, but to a longer-lived bound state which is formed in much less than $116 \mathrm{ps}$. The likelihood of this state being positronium-like will depend inter alia on the distribution of free volume in the material. X-ray diffraction and phase contrast electron microscopy show carbon black to consist of an incompletely ordered assembly of graphitic carbon planes, and the existence in carbon black of pores of atomic dimensions is confirmed by gas absorption measurements [6]. The x-ray data can be interpreted as giving a mean defect-free distance, which in Philblack A, for example, is about $1.5 \mathrm{~nm}$, leading to a defect concentration of order $3 \times 10^{20} \mathrm{~cm}^{-3}$. This figure is two orders of magnitude higher than the F-centre concentration sufficient to trap $10 \%$ [22] to $20 \%$ [23] of positrons in $\mathrm{KCl}$, and is also within a factor of two of the observed ESR spin concentration in this black [16], although insufficient data are available to determine whether or not this agreement is fortuitous.

It has thus been established that a) essentially all annihilation is occurring from a bound state, and b) the usual qualitative conditions for positronium formation are satisfied. It is hence not unreasonable to assume tentatively that positronium (or quasipositronium [24]) is formed with near unit probability in carbon black (although final confirmation of this assumption must await magnetic quenching and angular correlation experiments). Thus putting $p=1$ in (3), the radial diffusion coefficients of the bound state (whatever its nature) in the carbon blacks with the largest (SRF) and smallest (ISAF) particles must satisfy

$D_{\mathrm{SRF}}<1.3 \times 10^{-6} \mathrm{~cm}^{2} \mathrm{~s}^{-1} ;$
$D_{\mathrm{ISAF}}<1.1 \times 10^{-7} \mathrm{~cm}^{2} \mathrm{~s}^{-1}$,

where the appropriate values of $R$ from Table 1 have been used. These unusually low values may result from a) the fact, clearly apparent from high-resolution electron micrographs [6], that the graphitic 'planes' near the particle surface are curved and concentric with the surface, and would thus restrict radial diffusion; and possibly b) partial binding of the electron or positron to another atom, i.e., the state may be better described as quasi-positronium. Such states are not at present well understood [24]. Further evidence for the essential immobility of the bound positrons is given by the complete insensitivity of the lifetime to changes of over an order of magnitude in the unpaired spin concentration in the carbon blacks (Table 1). In GPF, the 
Table 3. Measured positron lifetime parameters

\begin{tabular}{|c|c|c|c|c|c|}
\hline \multirow[t]{2}{*}{ Sample } & & $I_{1}$ & $\tau_{1}[\mathrm{ps}]$ & $I_{2}[\%]$ & $\tau_{2}[\mathrm{~ns}]$ \\
\hline & & $\begin{array}{c}\text { (absolute } \\
\times 10^{-5} \text { ) }\end{array}$ & & & \\
\hline SRF & & 1.165 & $403 \pm 2.3$ & & \\
\hline GPF & & 1.178 & $398 \pm 2.3$ & - & - \\
\hline FEF & & 1.171 & $405 \pm 2.4$ & - & - \\
\hline HAF & & 1.159 & $395 \pm 2.4$ & $\ldots$ & - \\
\hline \multirow[t]{2}{*}{ ISAF } & & 1.156 & $400 \pm 2.4$ & - & - \\
\hline & {$[\mathrm{phr}[$} & {$[\%]$} & & & \\
\hline A & 100 & $83.6 \pm 0.19$ & $387+3.5$ & $16.4 \pm 0.19$ & $2.54 \pm 0.027$ \\
\hline B & 80 & $80.3 \pm 0.22$ & $377 \pm 3.7$ & $19.7 \pm 0.22$ & $2.48 \pm 0.024$ \\
\hline $\mathrm{C}$ & 60 & $76.3 \pm 0.24$ & $374 \pm 4.3$ & $23.7 \pm 0.24$ & $2.51 \pm 0.022$ \\
\hline D & 40 & $71.2 \pm 0.27$ & $374 \pm 4.6$ & $28.8 \pm 0.27$ & $2.52+0.019$ \\
\hline \multirow[t]{2}{*}{$\mathrm{E}$} & 20 & $61.0 \pm 0.35$ & $367 \pm 6.2$ & $39.0 \pm 0.35$ & $2.55 \pm 0.018$ \\
\hline & Black & {$[\%]$} & & & \\
\hline $\mathrm{F}$ & SRF & $74.9 \pm 0.26$ & $381 \pm 4.8$ & $25.1 \pm 0.26$ & $2.52 \pm 0.023$ \\
\hline $\mathrm{G}$ & GPF & $75.1 \pm 0.26$ & $378 \pm 4.6$ & $24.9 \pm 0.26$ & $2.55 \pm 0.023$ \\
\hline $\mathrm{H}$ & FEF & $76.9 \pm 0.24$ & $382 \pm 4.3$ & $23.1 \pm 0.24$ & $2.56 \pm 0.023$ \\
\hline I & $\mathrm{HAF}$ & $79.9 \pm 0.23$ & $368 \pm 4.1$ & $20.3 \pm 0.23$ & $2.50 \pm 0.025$ \\
\hline $\mathbf{J}$ & ISAF & $80.5 \pm 0.23$ & $387 \pm 4.3$ & $19.5 \pm 0.23$ & $2.51 \pm 0.026$ \\
\hline
\end{tabular}

typical distance between unpaired spins is $1 \mathrm{~nm}$, and an appreciable quenching rate would therefore be expected if the bound positrons were able to encounter these spins.

\subsection{Filled Rubber}

The Brandt and Paulin analysis, which leads to (1) and (2), is not directly applicable to the filled rubber results, because there is an appreciable probability of positrons thermalizing outside the carbon black particles (i.e. in the rubber), and there may be diffusion of positrons from the rubber to the black. An analytical solution for a modified model can, however, be obtained by the use of simplifying assumptions which are suggested by the experimental results and which are basically similar to those of Brandt and Paulin. The model consists of two concentric spheres of radii a and $\mathrm{b}$, the region $\mathrm{B}$ defined by $0 \leqq r \leqq a$ representing a carbon black particle, and $a<r \leqq b$ representing a region $R$ of rubber belonging to this particle in a sense to be explained. Positrons are assumed to thermalise with a probability density which is uniform in $B$, and also uniform, with a different value, in $R$; they annihilate at a rate $\gamma_{B}$ in $B$. In $R$, where positrons have diffusion coefficient $D$, a fraction $\phi$ of them annihilate at a rate $\gamma_{s}$ and the remainder at a lower rate $\gamma_{R}$. The simplifying assumptions are 1) no positrons escape from $B$, and 2) the surface of $B$ is an ideal sink for positrons arriving from $R$, which subsequently annihilate at a rate $\gamma_{B}$ in $B$. The data in Table 3 show that $\gamma_{B} \approx 6 \gamma_{R}$, so that assumption 2) should be at least qualitatively correct. The quantity $a$ is of course the radius of the carbon black particle, and $b$ is established as follows. Every carbon particle in the rubber defines a region, similar to a Wigner-Seitz cell, such that a positron in this cell has higher probability of diffusing to the particle within the cell than to any other. $b$ is chosen as an average radius of such a cell by equating the shell volume $4 \pi\left(b^{3}-a^{3}\right) / 3$ to the average volume of rubber per carbon particle $: b / a=f_{v}^{-1 / 3}$ where $f_{v}$ is the volume fraction of carbon black. The definition of the cell implies that at its boundary surface the normal derivative of the positron concentration vanishes. The boundary is therefore effectively impermeable, and only one cell need be considered. In place of (1) and (2) one obtains the following results

$$
\begin{aligned}
n(t)= & 6 C n_{R}(0) \sum_{v=1}^{\infty} Q_{v} \phi\left(\frac{1}{x_{v}^{2}}-\frac{\beta_{s}^{2}}{x_{v}^{2} \beta_{s}^{2}+g_{s}^{2}}\right) \\
& \cdot \mathrm{e}^{-\left(x_{v}^{2} \beta_{s}^{2}+1\right) y_{s} t} \\
& +6 C n_{R}(O) \sum_{v=1}^{\infty} Q_{v}(1-\phi)\left(\frac{1}{x_{v}^{2}}-\frac{\beta_{R}^{2}}{x_{v}^{2} \beta_{R}^{2}+g_{R}^{2}}\right) \\
& \cdot \mathrm{e}^{-\left(x_{v}^{2} \beta_{R}^{2}+1\right) \gamma_{R} t} \\
& +\left[n_{B}(0)+\Phi\right] \mathrm{e}^{-\gamma_{B} t},
\end{aligned}
$$

where the following abbreviations have been used (cf. (1) and (2)):

$$
\begin{aligned}
\Phi= & 6 C n_{R}(0) \sum_{v=1}^{\infty} Q_{v}\left[\phi \frac{\beta_{s}^{2}}{x_{v}^{2} \beta_{s}^{2}+g_{s}^{2}}\right. \\
& \left.+(1-\phi) \frac{\beta_{R}^{2}}{x_{v}^{2} \beta_{R}^{2}+g_{R}^{2}}\right]
\end{aligned}
$$

$\pm x_{v}$ are the roots of $\tan x=\frac{b}{b-a} x$

$C=\frac{a^{2}(b-a)}{b^{3}-a^{3}}, \quad \beta_{s}^{2}=\frac{D}{\gamma_{s}(b-a)^{2}}, \quad g_{s}^{2}=1-\frac{\gamma_{B}}{\gamma_{s}}$

and similarly for $\beta_{R}^{2}, g_{R}^{2}$,

$Q_{v}=\left[1-\frac{b(b-a)}{(b-a)^{2}+b^{2} x_{v}^{2}}\right]^{-1}$,

$n_{R}(0)$ is the number of positrons in $R$ at $t=0$, and similarly for $n_{B}(0)$.

The derivation of (4) and (5) will be found in the Appendix. Similar results are obtainable for plane and cylindrical geometries. For $D=0$ and $D=\infty$ one retrieves the expected results $n(t)=n_{R}(0)\left[\phi \exp \left(-\gamma_{s} t\right)\right.$ $\left.+(1-\phi) \exp \left(-\gamma_{R} t\right)\right]+n_{B}(0) \exp \left(-\gamma_{B} t\right)$ and $n(t)$ $=\left[n_{R}(0)+n_{B}(0)\right] \exp \left(-\gamma_{B} t\right)$, respectively. As with (1), a finite value of $D$ replaces each exponential characterising the region out of which diffusion occurs with an infinite sum of exponentials each of higher decay rate than the original. Interpretation of time spectra using 
(4) is however more difficult than using (1) because $g_{R}^{2}$ in (4) is negative in the present experiment, whereas $g^{2}$ in (1) was positive. In consequence, the range of decay rates derived from $\gamma_{R}$ will include (and that derived from $\gamma_{s}$ may include) the rate $\gamma_{B}$ associated with $\Phi$. When a decay rate in a summation is equal to $\gamma_{B}$, its coefficient becomes infinite, but is exactly cancelled by an identical coefficient of opposite sign in $\Phi$, and the total contribution to $n(t)$ remains well-behaved. Apart from such effects (which are essentially unobservable), the coefficients in the summations decrease rapidly with increasing $v$ because $(v-1) \pi<x_{v}<(v-1 / 2)$. The term $v=1$ in the second summation of (4) dominates the other long lifetime components provided that its decay rate is well separated from $\gamma_{B}$, which will clearly be true if it is to describe the data of Table 3. This conclusion has been verified by numerical calculation of the leading coefficients of (4) for a wide range of parameter values.

Extensive approximation is required before (4) can be compared with the results of a two-component time spectrum analysis. It is clear from Table 3 that the composition of the filled rubber samples affects principally the relative intensities of the components. Note also that the lifetime $\tau_{2}$ is constant within experimental error, which implies $\beta_{R}^{2} \ll 1$, and $\tau_{1}$ varies over a range of only $5 \%$, which implies $\gamma_{s} \approx \gamma_{B}$. Considering in addition that $\gamma_{B}^{-1}$ is about equal to the system FWHM time resolution, a suitable approximation is to assume that the exponential in (4) with the smallest decay rate can be identified with the observed long component, and that all other exponentials are unresolved and contribute to the short component only. This gives

$$
I_{2}=\frac{6 C n_{R}(0) Q_{1} \frac{1}{x_{1}^{2}}\left(1-\frac{x_{1}^{2} \beta_{R}^{2}}{x_{1}^{2} \beta_{R}^{2}+g_{R}^{2}}\right)(1-\phi)}{n_{R}(0)+n_{B}(0)} .
$$

Because $C$, the $Q_{v}$, the $x_{v}$ and $n_{R}(0) /\left[n_{R}(0)+n_{B}(0)\right]$ all depend on $a$ and $b$ only through the ratio $b / a$, the effect on $I_{2}$ of varying the particle size $a$ at constant loading (i.e. constant $b / a$ if all types of particle have the same density) should be given by the term $-x_{1}^{2} \beta_{R}^{2} /\left(x_{1}^{2} \beta_{R}^{2}+g_{R}^{2}\right)$. This is (minus) the fraction of positrons in the first spatial fourier component in $R$ which encounters the surface of $B$ before annihilating. In the present case this term is positive, because $g_{R}^{2}$ is negative and numerically greater than $x_{1}^{2} \beta_{R}$ (or there would not be a separate long lifetime), and these positrons therefore increase $I_{2}$ rather than $I_{1}$, the physical reason being that this means of annihilations is limited by the rate at which positrons arrive from $R$ and the number of positrons involved must therefore ultimately have the same time dependence as the number of positrons in $R$. Because $\partial \beta_{R}^{2} / \partial a \mid b / a=$ const $<0, I_{2}$ should be increased by decreasing the particle size at constant weight loading. (This conclusion depends essentially only on having $\gamma_{R} \ll \gamma_{B}$, and not on the particular geometry or approximation used. Such effects are characteristic of first-order processes in series, the best-known example being transient equilibrium of radioactive species [25]). The $I_{2}$ data in Table 3 for samples $\mathbf{F}-\mathbf{J}$ (which are listed in order of decreasing particle size see Table 2) do not conform to the above prediction.

Thus the dependence of $I_{2}$ on carbon black type cannot be explained on the basis of a model in which diffusion plays a dominant role, since the latter predicts an increase in $I_{2}$ with increasing particle surface area contrary to experimental observation. We return later to discuss this aspect of the experimental data and in the meantime explore the implications of a negligible value of the diffusion coefficient in conjunction with the data on samples A to $E$.

Suppose then that $D=0, \gamma_{s}=\gamma_{B}$, and that the probability for a positron to thermalize in carbon black is $k$ times that for an equal mass of rubber. Then the time spectrum for a sample with loading $f$ phr is proportional to (rubber spectrum) $+k f / 100 \cdot$ (carbon black spectrum). If the rubber spectrum (which was not observed) has a ratio of short to long component intensities $G$, then we should have

$\frac{1}{I_{2}}=(1+G) \frac{k f}{100}+(1+G)$.

A weighted least squares fit of (7) to the data for samples A to $\mathrm{E}$, using the standard deviations given in Table 3 , yields a $\chi^{2}$ value of 7.57 , which is less than the $5 \%$ level with 3 d.f. The fit can therefore be considered excellent (see Fig. 1) particularly in view of the fact that $\chi^{2}$ will have been increased by departures from the assumptions and by the use of the computed standard deviations of $I_{2}$, which err on the small side because they exclude any source of error other than counting statistics. The fit yields a value for $k$ of $2.5 \pm 0.09$.

There is, of course, another way in which we can obtain a value for $k$. The probability density $p(x)$ with which positrons from a radioactive source thermalize a distance $x$ from the entry surface in a homogeneous solid is often approximated $[26,27]$ by

$p(x)=\alpha \mathrm{e}^{-\alpha x}$

and Löbl and Paulin have shown [28] that for many solid elements and diatomic compounds, the absorption coefficient is well approximated by

$\alpha=(9.0 \pm 0.2) \varrho\left[1+(0.8 \pm 0.02) \ln Z_{\text {eff }}\right]$

where $\varrho$ is the density of the solid in $\mathrm{g} \mathrm{cm}^{-3}, Z_{\text {eff }}$ is a specially defined average atomic number of the solid, 


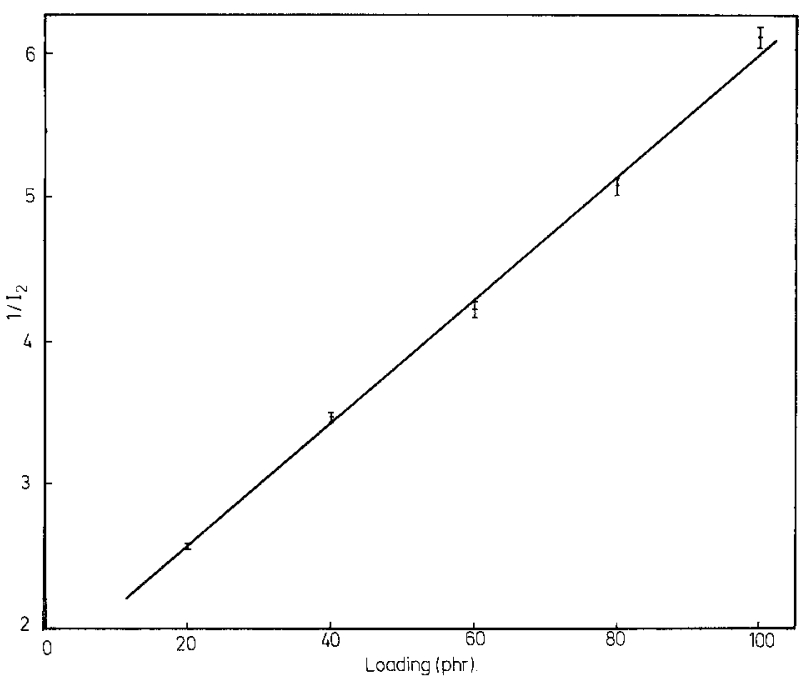

Fig. 1. $1 / I_{2}$ against Carbon Black loading for samples A-E (errors: 1 s.d.)

and the numerical constants are appropriate for ${ }^{64} \mathrm{Cu}$ positrons $\left(E_{\max }=0.65 \mathrm{MeV}\right.$; the ${ }^{22} \mathrm{Na}$ positrons used here have $E_{\max }=0.54 \mathrm{MeV}$ ). If (8) were unconditionally valid, the positron thermalization rate in a (homogeneous) volume element of dimensions $\ll \alpha^{-1}$ would be proportional to the local value of $\alpha$, and the thermalization rate per unit mass would therefore be proportional to the local value of $\alpha / \varrho$ a quantity which according to (9) depends only weakly on the nature of the solid. The value of $k$ calculated from (8) and (9) is in fact 1.07 , in clear disagreement with the experimental value. The exponential approximation (8) is, however, known (from electron data [29, 30], which are more extensive than positron data) to result fortuitously from the convolution of typical beta energy spectra with the highly non-exponential absorption curves for mono-energetic beta particles. The true value of $k$ will depend on the relative properties of rubber and carbon black for positrons with energies of order $10 \mathrm{eV}$, and at such energies a) the probability of formation of a bound state is important, and will depend sensitively on the material, and b) the positron directions of motion will already be randomized. There is thus no particular reason to expect (8) to predict the value of $k$ correctly for an inhomogeneous medium (although it should give a reasonable estimate of the total transmission through a thin inhomogeneous slice).

It is now clear that our assumption that diffusion effects as a function of loading are not observable in samples $A-E$ is correct, and this enables an approximate upper limit for the diffusion coefficient to be obtained. Some correction is necessary for the partial fusing together of some of the spherical carbon black particles, which reduces the available surface area.
Brandt has shown [26], for the model leading to (1) and (2), that the fraction of positrons which escape from a solid before annihilating is insensitive to the shape of the solid, and can be adequately calculated from the density and the specific surface area alone. A similar result should hold for (4) and (5), at least for the moderate values of $b / a$ which apply to the present samples, and the effective particle radius can thus be obtained from the surface area data of Table 1 . Diffusion effects should be largest for sample A, where the standard deviation of $I_{2}$ is $0.19 \%$ and a change of $1 \%$ in $I_{2}$ would therefore have been easily perceptible. Hence $\beta_{R}^{2} x_{1}^{2}<10^{-2}\left|g_{R}^{2}\right|$, and using the value $x_{1} \approx 1.36$ appropriate to the volume fraction 0.352 corresponding to a loading of $100 \mathrm{phr}$, one finds $\beta_{R}^{2}<2.8 \times 10^{-2}$ and thus $D<7.2 \times 10^{-5} \mathrm{~cm}^{2} \mathrm{~s}^{-1}$. Brandt and Paulin [17] found a value of $D=2.5 \times 10^{-4} \mathrm{~cm}^{2} \mathrm{~s}^{-1}$ for the much more confined environment of $\mathrm{MgO}$. (It should be noted incidentally that the non-observation of diffusion effects cannot be attributed to poor dispersion of the carbon black, since the previous study on these samples [10] showed that bound rubber increased with loading and that essentially all of the carbon black participated in the formation of bound rubber.)

We return now to the interpretation of data from samples $\mathrm{F}-\mathrm{J}$ and the observed decrease in $I_{2}$ with surface area. It is unlikely that this decrease in $I_{2}$ is due to differences in the filler particles in view of the constancy of the carbon black data of Table 3. Rather, the variation in $I_{2}$ is more likely associated with the rubber medium where, for example, no distinction has been made in the model between bound and unbound rubber [10]. If such considerations were to lead to an increase of $25 \%$ in the amount, $\phi$, of short rubber component in going from SRF to ISAF, this would account for the changes in $I_{1}$ and $I_{2}$. (The estimate assumes, of course, zero diffusion).

In a related ESR study on similar samples [16] a stable free radical signal has been observed in the filled rubber, the intensity of which increases linearly with the amount of bound rubber present. Coupled with the observation that bound rubber content increases with increasing surface area of the black, it is entirely reasonable that the presence of free radical species in the bound rubber would lead to a decrease in $I_{2}$. However, in the same ESR study there was no obvious correlation when rubber spin concentration was determined directly as a function of carbon black surface area. Whether this derives from an inability to sufficiently reduce the errors in spin assays as a function of surface area or is due to some more fundamental property of the filled materials is still an open question and as such our interpretation must be viewed as tentative. 


\section{Conclusions}

1) Evidence has been presented that annihilation of positrons in carbon black occurs principally from a bound state.

2) The variation in the long component intensity with loading has been satisfactorily explained.

3) Diffusion effects both in the carbon black and the rubber have been shown to be small and upper limits have been obtained for the diffusion coefficients of the postulated quasi-positronium state in carbon black and for positronium in polybutadiene.

4) A tentative explanation has been given for the observed decrease in long component intensity with increasing surface area of the carbon black filler in terms of the stable free radicals formed in the bound rubber component.

\section{Appendix}

\section{Diffusion of Unstable Particles in Concentric Spheres}

The derivation of (4) and (5) will be outlined. The method is similar to that used by Brandt and Paulin [17] to derive the equations reproduced here as (1) and (2). It is convenient to describe the problem in terms of diffusion of unstabie particles of unspecified nature.

Let $\tilde{p}(r, t)$ be the probability density for finding an unstable particle at position $\underline{r}$ and time $t$. It satisfies the diffusion-absorption equation

$\frac{\partial \tilde{p}}{\partial t}=-\gamma \tilde{p}+D \nabla^{2} \tilde{p}$

which is reduced by Danckwert's transformation $[31] \tilde{p}(\underline{r}, t)=p(r, t)$ $\exp (-\gamma t)$ to the diffusion equation for the probability density $p(r, t)$ of stable particles

$\frac{\partial p}{\partial t}=D \nabla^{2} p$

Under certain circumstances [31,32], solutions of (A.1) can be obtained from solutions of (A.2) for the same spatial boundary conditions. In order to have $D$ and $\gamma$ independent of $\underline{r}$ and $t$, we express the original problem as the superposition of three subproblems described by (A.1) with the following conditions:

I) Region $B(0 \leqq r \leqq a) ; D=0, \gamma=\gamma_{B}, \tilde{p}(\underline{r}, 0)=$ constant

II) Region $R(a<r \leqq b)$; $\mathrm{D} \neq 0, \gamma=\gamma_{s}, \tilde{\mathrm{p}}(\underline{\mathrm{r}}, 0)=\mathrm{constant}, \tilde{p}=0$ at $r=a$ and $\frac{\partial \tilde{p}}{\partial r}=0$ at $r=b$.

III) As II but with $\gamma=\gamma_{R^{*}}$

Sub-problems II and III (which describe fractions $\phi$ and $1-\phi$, respectively, of the particles originally present in $R$ ) are both derived from the same solution of (A.2), and for brevity only a single corresponding solution of (A.1) will be given, from which the solution of the original problem is easily recoverable.

The number of unstable particles in $R$ at time $t$ is given by

$n_{R}(t)=\int_{R} \tilde{p}(r, t) d V$,

where $d V$ is a volume element. Denoting the number of stable particles (in the transformed problem) remaining in $R$ at time $t$ by

$M(t)=\int_{R} p(\underline{r}, t) d V$, it follows that

$n_{R}(t)=M(t) \mathrm{e}^{-\gamma t}$

and that

$\frac{d n_{R}}{d t}=-\gamma n_{R}+\frac{d M}{d t} \mathrm{e}^{-\gamma t}$

in which the first term of the rhs represents decay of unstable particles, and the second their migration out of $R$ into $B$. Similarly for the number $n_{B}(t)$ of unstable particles in $B$ at time $t$ we have

$\frac{d n_{B}}{d t}=-\gamma_{B} n_{B}-\frac{d M}{d t} \mathrm{e}^{-\gamma t}$

the solution of which is readily found, by the use of the integrating factor $\exp \left(\gamma_{B} t\right)$, to be

$n_{B}(t)=n_{B}(0) \mathrm{e}^{-\gamma_{B} t}-\mathrm{e}^{-\gamma_{B} t} \int_{0}^{t} \frac{d M\left(t^{\prime}\right)}{d t^{\prime}} \mathrm{e}^{-\left(\gamma-\gamma_{B}\right) t^{\prime}} d t^{\prime}$

$M(t)$ is obtained from

$\frac{d M}{d t}=D \int_{S} \nabla p(r, t) \cdot d S$,

where $d S$ is an element of the surface $S$ separating $B$ and $R$. The stable-particle solution $p(r, t)$ in $R$ can be obtained as a special case of a solution given by Carslaw and Jaeger [33] for heat flow in a hollow sphere: let $\pm \alpha_{v}(v=1,2, \ldots)$ be the roots of $\tan [(b-a) \alpha]=b \alpha$, and define

$A_{v}=\frac{a\left(1+b^{2} \alpha_{v}^{2}\right)}{\alpha_{\nu}\left[\left(1+b^{2} \alpha_{v}^{2}\right)(b-a)-b\right]}$.

Then the solution of (A.2) which has $p(r, 0)=p_{0}$ (a constant) in $a<r \leqq b$ is

$p(r, t)=\frac{2 p_{0}}{r} \sum_{v=1}^{\infty} A_{v} \exp \left(-D \alpha_{v}^{2} t\right) \sin \left[(r-a) \alpha_{v}\right]$

which is easily seen to satisfy $p=0$ at $r=a$ and $\partial p / \partial r=0$ at $r=b$. Using (A.11) in (A.9) and integrating the result with respect to $t$, one obtains

$M(t)=8 \pi a p_{0} \sum_{v=1}^{\infty} \frac{A_{v}}{\alpha_{v}} \exp \left(-D \alpha_{v}^{2} t\right)$

and substituting this in to (A.8) yields for the total number $n(t)=n_{R}(t)$ $+n_{B}(t)$ of unstable particles

$$
\begin{aligned}
n(t)= & 8 \pi a p_{0} \sum_{v=1}^{\infty}\left(\frac{A_{v}}{\alpha_{v}}-\frac{A_{v} \alpha_{v} D}{D \alpha_{v}^{2}+\gamma-\gamma_{B}}\right) \exp \left[-\left(D \alpha_{v}^{2}+\gamma\right) t\right] \\
& +\left[n_{B}(0)+8 \pi a p_{0} \sum_{v=1}^{\infty} \frac{A_{v} \alpha_{v} D}{D \alpha_{v}^{2}+\gamma-\gamma_{B}}\right] \mathrm{e}^{-\gamma_{B} t} .
\end{aligned}
$$

Using the abbreviations

$$
\begin{aligned}
n_{R}(0) & =4 \pi\left(b^{3}-a^{3}\right) p_{0} / 3, \quad x_{v}=(b-a) \alpha_{v}, \\
C & =a^{2}(b-a) /\left(b^{3}-a^{3}\right), \\
Q_{v} & =\left[1-\frac{b(b-a)}{(b-a)^{2}+b^{2} x_{v}^{2}}\right]^{-1}, \quad \beta_{1}^{2}=\frac{D}{\gamma(b-a)^{2}}
\end{aligned}
$$

and $g_{1}^{2}=1-\gamma_{B} / \gamma,($ A.13) becomes

$$
\begin{aligned}
n(t)= & 6 n_{R}(0) C \sum_{\nu=1}^{\infty} Q_{v}\left(\frac{1}{x_{v}^{2}}-\frac{\beta_{1}^{2}}{x_{v}^{2} \beta_{1}^{2}+g_{1}^{2}}\right) \\
& \cdot \exp \left[-\left(x_{v}^{2} \beta_{1}^{2}+1\right) \gamma t\right] \\
& +\left[n_{B}(0)+6 n_{R}(0) C \sum_{v=1}^{\infty} Q \frac{\beta_{1}^{2}}{x_{v}^{2} \beta_{1}^{2}+g_{1}^{2}}\right] \mathrm{e}^{-\gamma_{B} t}:
\end{aligned}
$$


If the rhs of (A.14) is regarded as defining a function $f(t, \gamma)$, then the distinction between sub-problems II and III is correctly recovered by writing

$n(t)=\phi f\left(t, \gamma_{s}\right)+(1-\phi) f\left(t, \gamma_{R}\right)$,

which on expansion yields (4) and (5).

\section{References}

1. G.Kraus (ed.): Reinforcement of Elastomers (Interscience, London 1965)

2. G.Kraus: Rubber Chem. Technol. 38, 1070 (1965)

3. P.B.Stickney, R.D.Falb: Rubber Chem. Technol. 37, 1299 (1964)

4. W.C.Wake (ed.): Fillers for Plastics (Iliffe Publications, London 1970)

5. C.M.Blow: Polymer 14, 309 (1973)

6. J.B.Donnet, A.Voet: Carbon Black (Marcel Dekker, New York 1976)

7. S. Kaufman, W.P.Slichter, D.D.Davis: J. Polymer Sci. (A2) 9, $829(1971)$

8. T. Nishi: J. Polymer Sci. A (Polymer Phys.) 12, 685 (1974)

9. R.J.Roe, D.D.Davis, T.K.Kwei: Bull. Am. Phys. Soc. (Ser. 2) 15, 308 (1970)

10. J.O'Brien, E.Cashell, G.E.Wardell, V.J.McBrierty: Macromolecules 9, 653 (1976)

11. M.A.Waldrop, G.Kraus: Rubber Chem. Technol. 42, 1155 (1969)

12. G.Kraus, J.T.Gruver: J. Polymer Sci. (A2) 8, 571 (1970)
13. A.M.Gessler: Rubber Age (December 1969)

14. D.H.D.West, V.J.McBrierty, C.F.G.Delaney: Appl. Phys. 7, $171(1975)$

15. D.H.D.West: Nuc. Instr. Methods 136, 137 (1976)

16. E.Cashell, V.J.McBrierty: J. Material Sci. 12, 2011 (1977)

17. W.Brandt, R.Paulin: Phys. Rev. B5, 2430 (1972)

18. S.Berko: Private communication

19. W. Brandt, J. Reinheimer: Phys. Lett. 35 A, 109 (1971)

20. W.Brandt, J.Reinheimer: Phys. Rev. B2, 3104 (1970)

21. S. Mrozowski: Carbon 9, 97 (1971)

22. W.C.Mallard, F.H.Hsu: Phys. Lett. 38 A, 164 (1972)

23. A.Dupasquier: Nuovo Cimento Lett. 4, 13 (1970)

24. A. Dupasquier: Review paper presented at the 4th Inst. Conf. on Positron Annihilation, Helsingør, Denmark (August 1976)

25. W.E.Burcham: Nuclear Physics, an Introduction; 2 nd ed. (Longman, London 1973) p. 35

26. W.Brandt: Appl. Phys. 5, 1 (1974)

27. W.Brandt, R.Paulin: Paper presented at the 4th Int. Conf. on Positron Annihilation, Helsingør, Denmark (August 1976)

28. H.Löbl, R.Paulin : Conference paper as [27]

29. W.J.Price: Nuclear Radiation Detectors (McGraw-Hill, London 1958)

30. V.E.Cosslett, R. N.Thomas: Brit. J. Appl. Phys. 16, 779 (1965)

31. P.V.Danckwerts: Trans. Farad. Soc. 47, 1014 (1951)

32. J.Crank: The Mathematics of Diffusion; 2nd ed. (Oxford University Press, London 1975). Owing to a typographical error, the definition of Danckwerts' transformation on p. 329 of this reference is incorrect

33. H.S. Carslaw, J.C. Jaeger: Conduction of Heat in Solids; 2 nd ed. (Oxford University Press, London 1959) 